\title{
PLANEACIÓN E INCERTIDUMBRE*
}

Jorge Iván Gonzálezi

" DOI: https://doi.org/10.18601/01245996.v23n44.11. Recepción: 01-092020, modificación final: 28-10-2020, aceptación: 27-11-2020. Sugerencia de citación: González, J. I. (2021). Planeación e incertidumbre. Revista de Economía Institucional, 23(44), 233-248.

a Profesor Universidad Externado de Colombia, [jorgeivangonzalez29@ gmail.com], [https://orcid.org/0000-0003-4845-2690]. 


\section{Planeación e incertidumbre}

Resumen Este artículo examina los problemas de la planeación en condiciones de incertidumbre. Siempre es necesario planear, pero el ejercicio se puede realizar de muy diversas maneras. Aquí se establece la diferencia entre planeación basada en la probabilidad de clase y planeación basada en la probabilidad de caso, y se muestra que así esta última prevalezca, es inadecuada y fracasa. Un ejemplo de sus limitaciones es el mal manejo de la crisis causada por la COVID-19. Se propone, entonces, volver la mirada hacia la probabilidad de clase, que es necesaria y suficiente para plantear las opciones de planeación de manera adecuada. Aunque este ejercicio es pertinente, la planeación requiere un paso adicional, el de la elección colectiva, y es entonces ineludible preguntarse por la dimensión ética. El tamaño del Estado se resuelve en la arena política, y en las sociedades democráticas debe ser compatible con el ejercicio de la libertad.

Palabras clave: planeación, incertidumbre, política pública, tamaño del Estado, democracia; JEL: B41, D6, D80, H00, I1.

\section{Planning and uncertainty}

Abstract This article examines the problems of planning under conditions of uncertainty. Planning is always necessary, but exercise can be done in many different ways. Here the difference between planning based on class probability and planning based on case probability is established, and it is shown so that the latter prevails, it is inadequate and fails. An example of its limitations is the mishandling of the crisis caused by COVID-19. It is proposed, then, to turn our gaze towards class probability, which is necessary and sufficient to raise the planning options adequately. Although this exercise is relevant, planning requires an additional step, that of collective choice, and it is then inescapable to ask about the ethical dimension. The size of the state is resolved in the political arena, and in democratic societies it must be compatible with the exercise of freedom.

Keywords: planning, uncertainty, public policy, size of the State, democracy; JEL: B41, D6, D80, H00, I1.

\section{Planejamento e incerteza}

Resumo Este artigo examina os problemas de planejamento em condições de incerteza. O planejamento é sempre necessário, mas o exercício pode ser feito de muitas maneiras diferentes. Aqui, a diferença entre o planejamento com base na probabilidade da classe e o planejamento com base na probabilidade do caso é estabelecida e é mostrada para que esta prevaleça, é inadequada e falha. Um exemplo de suas limitações é o manejo incorreto da crise causada pelo COVID-19. Propõe-se, então, voltar nosso olhar para a probabilidade de classe, necessária e suficiente para levantar as opções de planejamento de forma adequada. Embora este exercício seja relevante, o planejamento requer uma etapa adicional, a da escolha coletiva, e então é inevitável perguntar sobre a dimensão ética. $O$ tamanho do Estado é resolvido na arena política e nas sociedades democráticas deve ser compatível com o exercício da liberdade.

Palavras-chave: planejamento, incerteza, políticas públicas, tamanho do Estado, democracia; JEL: B41, D6, D80, H00, I1. 
La nitidez extrema, la claridad, la certeza no se obtiene más que en detrimento de la integridad

Prigogine y Stenger (1983, p. 56).

T a pandemia de COVID-19 es una excelente oportunidad para Lreflexionar sobre las limitaciones de la planeación. Las proyecciones económicas usualmente están basadas en la probabilidad de caso. Y este es el fundamento de los procesos de planeación. Esta forma de mirar el futuro tiene numerosos inconvenientes, y conduce al fracaso. Entre otras razones, porque no reconoce la importancia de la catalaxia, y porque desconoce la relevancia de la incertidumbre. Se propone, entonces, planear sobre la probabilidad de clase. Se muestra que esta forma de proceder es necesaria y suficiente para el diseño de la política pública.

Los errores de la planeación basada en la probabilidad de caso se han hecho más evidentes a raíz de las dificultades causadas por la COVID-19. En la primera parte se explica el significado de la catalaxia y su relación con la planeación. En la segunda se explica la diferencia entre las proyecciones basadas en las probabilidades de clase y de caso. Después se relacionan los procesos de planeación con el tamaño del Estado. El nivel de intervención está marcado por la dimensión ética. A partir de algunos principios de la escuela de Frankfurt se muestra la posibilidad de construir una ciudadanía respetuosa de la libertad, aun en presencia de un Estado grande. Se concluye con algunas consideraciones sobre la planeación en estos tiempos de pandemia.

\section{CATALAXIA Y PLANEACIÓN}

La prevención de desastres exige planear aun sin conocer la fecha en que ocurrirá el fenómeno, ni su dimensión. De manera responsable se puede crear un fondo, que se refinancia cada año, en espera de que ocurra algún desastre. El desconocimiento del momento en que producirá el hecho no desvirtúa la necesidad de hacer los preparativos necesarios para compensar posibles daños. Siguiendo una argumentación similar, se puede decir, con certeza, que las medidas preventivas disminuyen las muertes por la COVID-19. Y también es evidente que si los sistemas de salud están preparados para eventos fortuitos, como una pandemia, los daños se minimizan. A pesar de que estos postulados se podrían aceptar con relativa facilidad, la práctica de los gobiernos parece desconocerlos. Entre otras razones, porque se pretende planear a partir de una casuística marcada por el 
positivismo. Se confía de manera excesiva en la capacidad de los métodos, y se desprecian lógicas de acción razonables, que tienen un alto componente de sentido común. Una vez se presenta la COVID-19, los gobiernos tratan de reaccionar con rapidez, pero estas acciones no están enmarcadas en una concepción de la protección social y económica de largo aliento. Además, no se están aprovechando las lecciones de la pandemia para replantear un orden institucional que desconoce la relevancia de la salud pública.

Los seres humanos planeamos. Imaginamos el futuro y con la información disponible en cada momento, buscamos avanzar hacia un estadio que consideramos superior al actual. Para Mises (1960) este es el principio rector de la acción humana. Y la comparación entre escenarios alternativos es la forma más básica de la planeación. E1 procedimiento que lleva a construir estos futuros imaginados escapa a la lógica racional. En palabras de Shackle (1972, p. 3), "el futuro es imaginado por cada ser humano, y el proceso de la imaginación es una parte vital del proceso de decisión". La imaginación es distinta de la razón. El futuro cabe en el espacio de la imaginación y no en las dimensiones del análisis racional, pues "lo racional es un mundo sin tiempo, seguro y de antemano reconciliado consigo mismo" (ibíd., p. xv). La lógica racional establece relaciones de causalidad ex-post.

Cuando el individuo toma decisiones que van a afectar su futuro, lo hace a partir de los múltiples escenarios imaginados. Su jerarquización está motivada por sentimientos y pasiones. Los intentos de ordenamiento lógico fracasan, y son claramente insuficientes para priorizar. La angustia permanente del ser humano radica en que

toda decisión es un compromiso. La alternativa finalmente escogida nunca permite un logro perfecto de los objetivos, pero se trata de la mejor solución posible bajo las circunstancias dadas (Simon, 1997, p. 5).

La lectura conjunta de Mises, Shackle y Simon permite sentar los siguientes principios: 1) De cara al futuro es inevitable escoger entre escenarios alternativos. 2) El individuo opta por la opción que, de acuerdo con su percepción, lo llevará a un estadio superior. 3) Esta jerarquización de alternativas es el motor de la acción humana. 4) El orden de prioridades necesariamente involucra la relación entre presente y futuro, y está marcado por la imaginación y no por la lógica racional. 5) El sujeto que decide es el único que puede definir las alternativas de su acción. 6) Cualquier decisión tiene consecuencias eternas en un proceso que es irreversible. 7) Los determinantes sub- 
jetivos de la acción humana son de muy diverso tipo, y pueden ser más o menos heterónomos ${ }^{1}$.

Los principios anteriores se aplican a la decisión individual. La planeación que hacen los gobiernos obliga a resolver de alguna manera las incompatibilidades entre las decisiones individual y colectiva ${ }^{2}$. En el campo de la ética es el tránsito, siempre conflictivo, entre la moral individual y la determinación de la norma social. Para la escuela de Frankfurt este proceso se logra mediante la acción comunicativa.

Frente a los complejos mecanismos de la planeación, que fácilmente llevan a lógicas dictatoriales, Mises (1960) y Hayek (1976) optan por confiar en las dinámicas endógenas de cada sociedad, que se expresan a través de procesos catalácticos. Sin necesidad de un planificador central, la dinámica que resulta de la interacción de los individuos va reconfigurando el orden colectivo.

\section{ES NECESARIO ESCOGER}

La decisión es inevitable. Cualquier mirada hacia el futuro abre un abanico infinito de alternativas. Frente a los múltiples escenarios imaginados es necesario elegir uno solo. Este tipo de escogencia puede realizarse en contextos muy limitados que no tienen nada que ver con la libertad de agencia. Uno de los imaginarios del esclavo es el triunfo de Lincoln y la abolición de las cadenas, otro es el asesinato del amo, otro es la rebelión, otro es el suicidio. En medio de sus limitaciones evidentes, puede terminar por aceptar la sumisión. Retomando la cita de Simon, estas opciones están condicionadas por las "circunstancias dadas". Se trata de escogencias en un contexto lleno de limitaciones. Es la frustrante decisión que toma el vendedor ambulante que tiene que elegir el punto de la ciudad en el que mejor podría ubicarse. Es consciente de las restricciones impuestas por su situación, o por las que crean las mafias que controlan el espacio urbano.

\section{LA BÚSQUEDA DE UN ESTADIO SUPERIOR}

La decisión que toma el individuo está marcada por su deseo de avanzar hacia un estadio superior. La interacción entre preferencias

1 E1 propio Kant (1983) aceptó que el imperativo categórico es un postulado ideal, y que la autonomía de la voluntad es un principio imposible de lograr en la vida real. La razón humana tiene la obligación de plantear como objetivo el mundo de los fines pero, al mismo tiempo, es consciente de su imposibilidad fáctica.

${ }^{2}$ Es el tema de la elección social (Arrow, 1963; Sen, 1979), y de la elección pública (Buchanan y Tullock, 1962). 
y meta preferencias va delimitando el sendero de la acción humana (Sen, 1973; González, 2000). Al observador, que actúa como censor, la decisión del otro le puede parecer censurable, pero desde la perspectiva del actor, estas opciones son consecuentes con su imaginario de lo que podría ser un estadio superior. Finalmente, lo que importa es el juicio subjetivo sobre lo bueno, que es el motivo final de la acción del individuo. El punto de vista del censor termina siendo irrelevante.

\section{LA JERARQUIZACIÓN DE ALTERNATIVAS}

El individuo se ve en la obligación de jerarquizar entre todos los imaginarios posibles. Sin este ordenamiento la acción es imposible. El papel de la lógica racional pasa a un segundo plano, y adquieren relevancia las premisas y los juicios valorativos.

En tal caso, la razón sólo funcionará después de que haya sido provista de un adecuado surtido de información o de premisas. Si la razón existe para aplicarse al descubrimiento y elección de vías de acción, entonces esa información incluye, por lo menos, una serie de deber-ser, o de valores por obtenerse, y una serie de es, o de hechos referentes al mundo en el que va a tener efecto la acción. Cualquier intento por justificar, por medio de la lógica, estos deber-ser y es, simplemente conducirá a un retroceso hasta nuevos deber-ser y es que se postulan de manera similar (Simon, 1989, p. 16).

\section{PRESENTE y FUTURo}

La decisión se realiza en un contexto intertemporal. No es posible congelar el tiempo a través de recursos arbitrarios como el estado estacionario. El futuro no se puede entender como una secuencia lineal, en la que las predicciones y las certezas son posibles. Y esta dimensión de futuro significa que los imaginarios se van modificando segundo a segundo. Por tanto, la decisión no puede ser consistente a lo largo del tiempo (Elster, 1983). Las condiciones de estado estacionario, que se desprenden del principio de correspondencia de Samuelson (1983), se siguen utilizando en los análisis económicos. En condiciones de estado estacionario el presente es igual al futuro. El tiempo se congela. Y, en sentido estricto, el modelo deja de ser dinámico (Hicks, 1989).

\section{LA DECISIÓN DEL SUJETO ES SOLITARIA}

El individuo se enfrenta absolutamente solo al futuro. El ordenamiento de los imaginarios es de su exclusiva competencia. Es el drama inherente a toda acción humana. Aun en condiciones de alienación y de heteronomía, la persona termina cargando con las consecuencias 
de su acción, así que la opinión, o el poder opresivo de los demás, no borra la sensación de soledad. En palabras de Prigogine y Stenger (1983, p. 23): "se ha roto la antigua alianza; el hombre sabe ahora que está solo en la indiferente e inconmensurable inmensidad del universo del cual surgió por azar".

\section{LAS CONSECUENCIAS DE LA DECISIÓN SON ETERNAS E IRREVERSIBLES}

Las decisiones de las personas tienen consecuencias eternas y, además, son irreversibles. Las secuencias derivadas de la decisión de tener o no tener un hijo se mantendrán por siempre, y este tipo de decisión no se puede echar para atrás. En los aspectos relacionados con el clima, tal y como lo advierte el primer informe al Club de Roma, la irreversibilidad de los impactos que tiene la actividad humana en el planeta es una de los factores más preocupantes de la sostenibilidad ambiental (Meadows, 1972). El informe muestra que hay una clara asimetría entre las implicaciones de largo plazo de la decisión humana, y el horizonte miope de los sujetos, que a duras penas incorpora una o dos generaciones hacia adelante. Los asuntos relacionados con el cambio climático requieren que las opciones respondan a enfoques de larguísimo plazo (150-200 años), y esta visión no es compatible con la visión de corto plazo del sujeto individual. Esta asimetría, que es sustantiva, únicamente se podría compensar con un ordenamiento institucional, que ponga en primer lugar los asuntos ambientales.

Desde el punto de vista matemático, la reversibilidad supone que los movimientos a lo largo del tiempo se pueden devolver, así que $t \rightarrow-t$. De manera similiar, las velocidades también se pueden invertir y $v \rightarrow-v$. El estado estacionario, tal y como se emplea en economía, supone la reversibilidad.

Más allá del estado estacionario, la dinámica tal y como la conciben Keynes (1976), Hicks (1989) y los teóricos austriacos (Mises, 1960; Mitchell, 1927; Böhm-Bawerk, 1890), vincula el presente y el futuro, y no acepta la reversibilidad. Hay dinámica, dice Hicks, porque el pasado incide en el futuro.

Y la irreversibilidad guarda una estrecha relación con las características del agente y con su disponibilidad de información. La misma persona en dos momentos del tiempo, tiene conjuntos de información diferentes y, por tanto, su decisión no va a ser la misma. Las condiciones exógenas van cambiando segundo a segundo, y con ellas la percepción del sujeto (Elster, 1983). Por tanto, ningún segundo es repetible. 


\section{LOS DETERMINANTES SUBJETIVOS DE LA ACCIÓN SON DE DIVERSOS TIPOS}

$\mathrm{E} 1$ individuo organiza sus preferencias de acuerdo con los prejuicios valorativos. La visión del mundo es diferente para el fotógrafo, el arquitecto, el cantante, el economista, el poeta... Y la decisión de cada persona está marcada por su escala valorativa. De nuevo, en numerosas situaciones, el sujeto está condicionado, y sus opciones no son autónomas. Los determinantes contextuales importan. Commons (1990) introduce la categoría de "persona institucionalizada" para mostrar que las opciones del individuo están marcadas por la geografía, la cultura, la religión, la lengua, la educación, la familia, etc. Y si los márgenes de acción son muy pocos, la voluntad es heterónoma. Si son relativamente amplios, la decisión se puede considerar autónoma.

Para contrarrestar la mirada de corto plazo se requiere que haya procesos que permitan pasar de la elección individual a la colectiva. Para superar la diversidad de opiniones se tiene que proporcionar algún mecanismo de elección colectiva. $\mathrm{E} 1$ interés privado no logra resolver problemas colectivos complejos. Smith (2002, p. 512) considera que la crisis energética que se presentó en California en 2000/2001 tiene mucho que ver con la desregulación. Las empresas privadas, dice Smith, no tienen la capacidad de actuar en función de los largos plazos que requiere la conservación de las cuencas. Los equilibrios financieros anuales, que preocupan a los privados, no les permiten actuar en función de la temporalidad de los procesos ambientales.

\section{DOS MODALIDADES DE PLANEACIÓN: PROBABILIDAD DE CLASE Y PROBABILIDAD DE CASO}

Aun si se aceptan las dinámicas endógenas de los procesos inmersos en la catalaxia, es necesario ir rediseñando los futuros imaginados. Siempre se requiere alguna forma de coordinación colectiva, y en la realidad se construye algún mecanismo que permita pasar de la preferencia individual a la colectiva. Estos diseños van desde la complejidad de la decisión de una pareja, hasta las dificultades intrínsecas a las decisiones que se toman dentro de la familia, la empresa, la universidad, la iglesia, la sociedad... En las democracias se recurre al voto, y las opciones electores son muy diversas, según las diversas modalidades de ordenamiento institucional.

La pandemia de COVID-19 ha puesto en evidencia la falta de planeación. Y una explicación de este fracaso está relacionada con el afán de programar sobre la probabilidad de caso, y no sobre la proba- 
bilidad de clase (Mises, 1960). Y este error metodológico es notorio en los ejercicios de planeación económica.

La planeación fundada en la probabilidad de clase parte de aseveraciones que son bastante ciertas, y algunas de ellas son irrebatibles. Por ejemplo: “en Bogotá habrá un temblor”, "el río Magdalena se inundará", "el costo de las mercancías físicas aumenta cuando las vías son de mala calidad", "los sistemas de riego contribuyen a mejorar la productividad rural", "la ciencia y la tecnología favorecen el crecimiento", "la protección de las cuencas contribuye a la sostenibilidad ambiental", "en general, los jóvenes viven más que los viejos", "la prevención en salud es menos costosa que la atención clínica”.

La planeación basada en la probabilidad de caso supone que el futuro es previsible en detalles como, por ejemplo, "en 2031, el crecimiento del PIB será del 3,3\%", “el dólar estará a 4.506 pesos”, "la tasa de interés será del 7\%", " "al terminar el año la inflación será del 3\%”3. Estos pronósticos no se cumplirán. En todos los países es una práctica usual que los ministerios de hacienda, los centros de investigaciones y las calificadoras de riesgo hagan este tipo de proyecciones. En vez de reconocer el fracaso de las estimaciones, se insiste en repetir ese ejercicio (Taleb, 2007). A pesar de su fragilidad intrínseca, la planeación sobre la probabilidad de caso sigue primando.

La probabilidad de clase es necesaria y suficiente para definir las prioridades de la política pública. Es necesaria porque la política pública exige un cierto nivel de planeación. Y la jerarquización de las acciones requiere que haya principios relativamente ciertos, como "la prevención en salud es menos costosa que la atención clínica”. Si en Colombia se hubiera aceptado este postulado elemental, la seguridad social tendría mayor cobertura y calidad. Y no se pone en práctica porque no obstante su sencillez, implica una transformación radical del modo de funcionamiento de las entidades promotoras de salud (EPS). Las dificultades financieras del sistema de salud serían menores si se le hubiera dado una mayor relevancia a la prevención ${ }^{4}$.

Otro ejemplo de probabilidad de clase. Hay bastante consenso en que "la ciencia y la tecnología favorecen el crecimiento". Y aunque no se desvirtúa la pertinencia de este postulado, el presupuesto para el Ministerio de Ciencia y Tecnología es ridículo.

${ }^{3}$ Estas cifras corresponden a las proyecciones del Marco Fiscal de Mediano Plazo (Ministerio de Hacienda 2020).

${ }^{4}$ Esta recomendación ha sido reiterada por la Comisión del Gasto (2017). 
Si “los jóvenes viven más que los viejos" se tienen que diseñar mecanismos para que las personas de menor edad comiencen a ahorrar desde temprano, y permitan la sostenibilidad financiera del sistema.

La probabilidad de clase es suficiente porque of rece la información adecuada para tomar decisiones de acción colectiva. Cabe advertir que este conocimiento no resuelve el problema de la elección colectiva, sino que permite poner en evidencia el abanico de alternativas. En la esfera política es donde se decide si los dineros se destinan a la conservación de las cuencas, o al estímulo del transporte eléctrico. La probabilidad de clase es suficiente para explicitar ambas necesidades, pero su jerarquización corresponde al espacio de lo político.

Estos ejemplos muestran que las afirmaciones correspondientes a la probabilidad de clase, of recen la información necesaria y suficiente para tomar decisiones. Una de las razones que explican las dificultades de numerosos países para enfrentar la crisis de la COVID-19 ha sido la debilidad estructural de sus sistemas de salud, y esta limitación puede estar relacionada con el desprecio a las evidencias ofrecidas por la probabilidad de clase.

La probabilidad de clase permite, además, que actúe la razón pública (Habermas, 1962, 2008). Y este proceso es compatible con la catalaxia. La coordinación social tiene un fundamento discursivo. Este es el punto de partida de la teoría de la acción comunicativa.

La sociedad abre el espacio para que los individuos participen de múltiples maneras, en función de sus preferencias y visiones del mundo. La democracia participativa permite que los ciudadanos responsables transformen su poder social en autoridad racional. Para que ello sea posible el ordenamiento de la sociedad obliga a reducir, sobre todo, la desigualdad económica. Las modalidades de participación van más allá de las formas parlamentarias, y la protesta callejera adquiere relevancia (Wiggershaus, 1995, p. 550).

Los ciudadanos han de ejercitar activamente sus derechos de comunicación y sus derechos de participación, y no sólo en función de su propio interés bien entendido, sino orientándose al bien de todos. Esto exige la complicada y frágil puesta en juego de una motivación, que no es posible imponer por vía legal. De ahí que las virtudes políticas sean esenciales para la existencia de una democracia. Esas virtudes son un asunto de la socialización, y del acostumbrarse a las prácticas y a la forma de pensar de una cultura política traspasada por el ejercicio de la libertad política y de la ciudadanía (Habermas, 2008, p. 31).

De manera general, la catalaxia supone que los infinitos puntos de vista y la multiplicidad de actividades humanas encontrarán la forma de llegar a un orden espontáneo. En las democracias, el resultado 
colectivo tiene que estar inspirado en la razón pública. Las creencias y las pasiones individuales animadas por las "virtudes políticas" deben conducir a la formación de una cultura que garantice la libertad y el ejercicio de la ciudadanía. En opinión de Habermas, este proceso es "complicado" y "frágil".

El tribunal de la opinión, decía Bentham (1995), tiene tres componentes: información, deliberación y decisión. Para que el tribunal pueda operar de manera adecuada se requiere que haya información oportuna, y que se creen espacios adecuados para la deliberación y la decisión. La conversación es un elemento sustantivo del razonamiento público. Gracias al diálogo la opinión del otro puede ser tenida en cuenta ${ }^{5}$.

La probabilidad de caso no es un instrumento útil de planeación. Se debería rechazar porque al desconocer la incertidumbre, busca reglas casuísticas que desconocen la complejidad de las interacciones humanas. La probabilidad de caso se relaciona directamente con el comportamiento del individuo, pero es inaceptable para la planeación que guía la acción colectiva. La persona imagina el futuro con afirmaciones de este tipo: "mañana me encuentro con Isabel a las 7 pm", "Miguelito se casa el 15 de enero siguiente a las 3 pm", "me gradúo el 20 de noviembre a las 5 pm", "nos entregan el apartamento el 12 de octubre", "compro la bicicleta hoy porque mañana sube de precio". Estos ejemplos de probabilidad de caso son el motor de la acción individual. $\mathrm{Y}$ aunque es frecuente que no se cumplan, son una guía inevitable para la toma de decisiones cotidianas del sujeto. Pero desde la perspectiva de la acción colectiva, y desde la lógica de la razón pública, es inaceptable planear sobre la probabilidad de caso.

$\mathrm{E} 1$ asunto no es menor porque tiene serias implicaciones políticas. En el plan de desarrollo Pacto por Colombia, Pacto por la Equidad (Congreso de la República, 2019), el gasto está sometido a las proyecciones del Marco Fiscal de Mediano Plazo. Ello significa que las proyecciones casuísticas -basadas en la probabilidad de caso- del ministerio de $\mathrm{Ha}$ cienda terminan siendo el punto de referencia para decidir cuánto se

\footnotetext{
5 "la acción comunicativa me permite en un primer momento, el de comprensión de otras perspectivas del mundo, de otras culturas y formas de vida, reconocer la contingencia y la facticidad de mis puntos de vista, abrirme a la multiplicidad de lo diverso y aceptar la heterogeneidad de posiciones, visiones del mundo y concepciones del bien. La posibilidad de comprender a otros implica mi auto reconocimiento. Pero comprender a otros no significa tener que estar de acuerdo con ellos" (Hoyos, 1993, p. 7).
} 
gasta en cada sector ${ }^{6}$. Así se decide, en forma discrecional y arbitraria, que cierta inversión no es relevante porque su financiación amenaza la "estabilidad fiscal". Una vez actúa la dictadura de la regla fiscal, el debate razonado desaparece del panorama, y la opción última queda en manos de una reducida élite tecnocrática. El permanente error de las proyecciones obliga a replantear nuevamente las estimaciones, de modo que la sociedad queda sometida al poder de la ideología tecnocrática. $Y$ en esta secuencia de errores no se sanciona a quienes se equivocan (Taleb, 2007).

\section{LA ÉTICA Y EL TAMAÑO DEL ESTADO}

Las reflexiones sobre la planeación obligan a pensar en el tipo y el tamaño del Estado. Brennan y Buchanan (1977), así como Hayek (1991) y Mises (1985), le temen a gobiernos que por su tamaño y poder de intervención, terminen minando la autonomía de las personas. Un Estado, como el que se desprenden del Leviatán de Hobbes (1651), dicen Brennan y Buchanan, aniquila la libertad que exige el mercado. E1 poder leviatánico consume libertad a cambio de seguridad, y termina convertido en un monstruo que aniquila al sujeto.

En 1947, para los miembros de la Société du Mont-Pèlerin, era impensable que pudieran convivir, en el mismo espacio, una alta dosis de intervención del Estado y el reconocimiento del mercado como validador, con márgenes de libertad razonables. Desde Mont-Pèlerin, Mises y Hayek imaginaron que el poder estatal conducía inexorablemente a la dictadura, y no concibieron sociedades en las que pudieran convivir grandes estados con un espacio de libertad razonable (p. ej., Canadá, Noruega, Suecia, Islandia, Nueva Zelanda). Estos países han logrado altos niveles de protección y de seguridad social, sin menoscabar las libertades individuales. Y este proceso ha ido a la par con exigentes políticas distributivas.

El tamaño del Estado es un asunto que se define en el espacio de la ética, y esta dimensión es irremplazable. La visión comprehensiva de la ética y de la moral suele ser desconocida por los economistas,

\footnotetext{
6 "Artículo 5. Consistencia fiscal del plan nacional de inversiones públicas. El valor total de los gastos que se realicen para la ejecución del presente plan financiados con recursos del Presupuesto General de la Nación, no podrán superar en ningún caso el monto de los recursos disponibles de conformidad con el Plan Macroeconómico y el Marco Fiscal de Mediano Plazo" (Congreso de la República 2019).

7 En el Marco Fiscal de Mediano Plazo de 2019 se decía que en 2020 la economía crecería al 4\%. E1 error de proyección será significativo.
} 
que pretenden establecer una frontera artificial entre la técnica y la política. Los procesos de elección colectiva, en tanto se trata de escoger entre estados del mundo (Arrow, 1963), obligan a transformar la moral individual en norma colectiva.

Para la democracia sólo pueden reclamar legitimidad las leyes, que al establecerse legalmente en un proceso discursivo pueden encontrar acuerdo entre los asociados. Mientras el principio moral opera en el nivel de una racionalidad interna de las normas, el de su validez universal y categórica, el principio de la democracia se ocupa de la institucionalización externa de las normas: es decir de todo aquello que tiene que ver con los presupuestos y desarrollos discursivos de los acuerdos, a partir de los cuales se establecen leyes, se las hace aceptables y viables su cumplimiento (Hoyos 1993, p. 10).

La frase de Hoyos expresa claramente la forma como la acción comunicativa, el "proceso discursivo", logra resolver el paso de la moral individual a la norma de validez universal y categórica. El imperativo kantiano no está dado, sino que se construye a través del diálogo y de la comunicación. Se podría afirmar que el mínimo moral es una idea compartida de la justicia.

La disyuntiva simplista entre fallas del mercado y fallas del Estado, que es frecuente en economía, desvirtúa la relevancia de la moral y de la ley en la reconfiguración permanente del quehacer de lo público.

La planeación basada en la probabilidad de caso niega, de entrada, las bondades de la acción comunicativa porque obliga a aceptar la verdad de quien hace la proyección. Se podría argumentar que el poder del ministro de hacienda le ha sido concedido por la ley, y por el "desarrollo discursivo de los acuerdos". Y aun si en gracia de la discusión se aceptara esta premisa, el argumento queda invalidado si se reconoce que en las sociedades democráticas la acción comunicativa se reconfigura cada día. Desde esta perspectiva es inaceptable que un instrumento como el Marco Fiscal pretenda regir la política económica hasta 2031.

El tamaño raquítico del Estado colombiano es la expresión de una democracia limitada, que no ha sido capaz de avanzar hacia una tributación progresiva, y que niega las bondades intrínsecas de la inclusión y de la acción comunicativa.

\section{PLANEACIÓN EN TIEMPOS DE LA COVID-19}

Es inaceptable que en medio de la incertidumbre que acompaña la evolución de la pandemia, se pretenda planear a partir de la probabilidad de caso. Y, peor aún, que estas proyecciones arbitrarias se im- 
pongan como determinantes de las decisiones de la política pública. En los documentos de las entidades internacionales y nacionales abundan las cifras exactas sobre el comportamiento futuro del PIB, del empleo, de la inflación, del petróleo, etc. En todas las estimaciones se supone un conocimiento bastante preciso de las consecuencias de la pandemia. Estos ejercicios, como la regla fiscal, son el símbolo de un positivismo ingenuo, que mantiene la lógica propia de la escuela de Saint Simon. Se pretende organizar la sociedad de manera discrecional. Es la aplicación acrítica de la ingeniería al manejo de la sociedad. $\mathrm{Y}$ este error fatal, al decir de Hayek, tiene consecuencias negativas.

Al recordar a Kant, Hoyos (1978) afirma: "la auténtica política le es imposible dar un solo paso sin haber rendido antes homenaje a la moral". El pensar en público favorece el proceso deliberativo de la sociedad. Pensar en público es un acto intrínsecamente responsable.

E1 conflicto carece de solución si cada una de las partes insiste en la rectitud de lo que hace basado en la 'verdad' de sus principios éticos, porque puede suceder que los principios del oponente sean diferentes e igualmente justificados, al menos en su contexto. Se hace necesario acudir entonces a un principio de acción de orden diferente, es decir a un principio mínimo moral: éste exige que las personas puedan responder de sus acciones con principios deontológicos que no puedan dejar de ser aceptados recíprocamente en general por todos los afectados por ellos (Hoyos 2004, p. 6).

La acción comunicativa no es perfeccionista. Y la economía tiene que renunciar a su pretensión de conocer el futuro, con el detalle de la probabilidad de caso. E1 discurso tiene que ser interdisciplinar. Todos los saberes son parciales, y la economía no puede pretender explicar por sí sola la complejidad de las relaciones de mercado que, ante todo, son interacciones entre personas. La construcción de un discurso que tenga en cuenta la dimensión del otro, únicamente es posible si la planeación está fundada en la probabilidad de clase.

\section{REFERENCIAS BIBLIOGRÁFICAS}

Arrow, K. (1963). Social choice and individual values [1951]. Nueva York: Wiley.

Bentham, J. (1995). A fragment on government [1776]. Cambridge: Cambridge University Press.

Böhm-B., E. (1890). Capital and interest. A critical history of economic theory [1884]. Londres: McMillan.

Brennan, G, y Buchanan, J. (1977). Towards a tax constitution for leviathan. Journal of Public Economics, 8(3), 255-273.

Buchanan, J. y Tullock G, (1962). The calculus of consent. Logical foundations of constitutional democracy. Ann Arbor: University of Michigan Press. 
Comisión del Gasto y la Inversión Pública. (2017). Informe final. Bogotá: CGIP, Ministerio de Hacienda, Fedesarrollo.

Commons, J. (1990). Institutional economics. Its place in political economy [1934], 2 vol. New Brunswick, N.J.: Transactions Publishers.

Congreso de la República. (2019). Texto definitivo plenaria Cámara al Proyecto de Ley no. 311 Cámara - 227 Senado “por el cual se expide el Plan Nacional de Desarrollo 2018-2022 Pacto por Colombia, Pacto por la Equidad. Bogotá: Cámara de Representantes.

Elster, J. (1983). Uvas amargas. Sobre la subversión de la racionalidad. Barcelona: Peninsula.

González, J. I. (2000), La ilegalidad, los metaordenamientos y las metapreferencias. Revista de Economia Institucional, 2(2), 37-52.

Habermas, J. (1989). The structural transformation of the public sphere [1962]. Cambridge: MIT Press.

Habermas, J. (2008), Sobre las bases morales prepolíticas del Estado liberal I: razón secular y religión en el Estado moderno. En J. Habermas y J. Ratzinger (eds.), Entre razón y religión. Dialéctica de la secularización (pp. 24-45). Madrid: Fondo de Cultura Económica.

Hayek, F. von. (1991). The road to serfdom [1944]. Londres: Routledge.

Hayek, F. von. (1976). Law, legislation and liberty. The mirage of social justice, v. 2. Chicago: University of Chicago Press.

Hicks, J. (1989). Métodos de economía dinámica [1985]. México DF: Fondo de Cultura Económica.

Hobbes, T. (1651). Leviathan or the matter, forme and power of a Commonwealth ecclesiastical and civil. Londres: Green Dragon.

Hoyos, G. (1978). E1 problema de la libertad humana en Kant. Ideas y Valores, 51-52, 57-74.

Hoyos, G. (1993). Ética discursiva, derecho y democracia. Análisis Político, 20, 5-19.

Kant, I. (1983). Fundamentación de la metafísica de las costumbres [1785]. Buenos Aires: Porrúa.

Keynes, J. M. (1976). Teoría general de la ocupación, el interés y el dinero [1936]. México DF: Fondo de Cultura Económica.

Meadows, D., Meadows, D. et al. (1972). Los limites del crecimiento. México DF: Fondo de Cultura Económica.

Ministerio de Hacienda y Crédito Público. (2020). Marco fiscal de mediano plazo 2020. Bogotá: Ministerio de Hacienda.

Mises, L. von. (1960). La acción humana. Tratado de economía [1949], 2 vol. Valencia: Fundación Ignacio Villalonga.

Mises, L. von. (1985). Omnipotent government. The rise of the total State and total war [1969]. Grove: Libertarian Press.

Mitchell, W. C. (1927). Business cycles: The problem and its setting [1913]. Nueva York: NBER.

Prigogine, I. y Stenger, I. (1983). La nueva alianza. Metamorfosis de la ciencia [1979]. Madrid: Alianza.

Samuelson, P. (1983). Foundations of economic analysis [1947]. Cambridge: Harvard University Press.

Sen, A. (1973). Behaviour and the concept of preference. Economica, 40(159), 241-259. 
Sen, A. (1979). Collective choice and social welfare [1970]. Ámsterdam: Elsevier.

Shackle, G. L. S. (1992). Epistemics and economics. A critique of economic doctrines [1972]. New Brunswick, N. J.: Transaction Publishers.

Simon, H. (1997). Administrative behavior. A study of decision-making processes in administrative organization [1945]. Nueva York: Free Press.

Simon, H. (1989). Naturaleza y limites de la razón humana [1983]. México DF: Fondo de Cultura Económica.

Smith, V. (2002). Constructivist and ecological rationality in economics, Nobel Lecture. Fairfax, VA: George Mason University.

Taleb, N. (2007). The black swan. The impact of the highly improbable. Nueva York: Ramdon House.

Wiggershaus, R. (1995). The Frankfurt School. Its history, theories and political significance [1986]. Cambridge: Polity Press. 